\title{
Speed-Optimized Simulation Models for Rapid Performance Evaluation of Heating and Energy Management Systems
}

\author{
James Allan ${ }^{1}$, Max Boegli ${ }^{2}$, Andrew Bollinger ${ }^{1}$, Pierre-Jean Alet ${ }^{2}$, Matthias Wiget ${ }^{3}$ \\ ${ }^{1}$ Empa, Dubendorf, Switzerland \\ ${ }^{2}$ CSEM, Neuchatel, Switzerland \\ ${ }^{3}$ Eturnity, Chur, Switzerland
}

\begin{abstract}
State-of-the-art building simulation programs require detailed user input and long simulation times to capture the dynamic behavior of the building envelope and its integrated energy systems. A simplistic model will often be chosen for its speed but at a cost of reduced accuracy. This study uses Support Vector Regression to generate a trained model that captures the complexity of system models created in Modelica, but yields faster results. This work is unique because it attempts to capture the non-linear dynamics that occur due to feedback loops present in a system with multiple technologies and controllers. In this paper the results of three case studies using single zone buildings are presented. An annual heating profile at 15 min resolution, was predicted $13 \mathrm{x}$ faster than the detailed simulation, with an RMSE of $2.5 \mathrm{~kW}$ and overall error of $0.12 \%$; however the performance of the trained model relied on the quality and relevance of the input data. This paper communicates the process and considerations of using Support Vector Regression to predict the performance profile of a building using a model trained on the results of detailed simulation.
\end{abstract}

\section{Introduction}

Building Performance Simulation (BPS) is typically used to predict the heating and cooling demands of a building. The peak load is then used to size the heating and cooling systems (Burdick, 2011). By sizing for peak demand, the designed system will meet the demand at any time of the year; however, it may lead to a system that is under utilized for most of the year, particularly in climates with high seasonal variation. With increased integration of intermittent renewables, for example wind and solar, an optimal solution is one where the supply and demand profiles match. The challenge for BPS is to capture the coupling between energy generation, energy demand and energy usage.

Next generation BPS tools are actively being developed through collaborative efforts by the International Building Performance Simulation Associa- tion (IBPSA) working group (IBPSA, 2017). The tools consist of Modelica Libraries containing objectoriented building and envelope components that can be reused and assembled to investigate novel system configurations. Despite the large effort into expanding the capabilities of BPS, there is still a high cost in computation when simulating systems that consist of many components and sub-systems. For applications where multiple options and configurations need to be simulated rapidly, the processing time can be prohibitively long.

The need for a fast and accurate approach to building performance simulation was voiced by Eturnity AG, Switzerland, who wish to ultimately implement the approach into their online platform (Eturnity, 2019). To be practical for their purposes, the target simulation for each configuration is less than 30 seconds with the annual energy consumption deviating no less than $20 \%$ from the detailed simulation.

Studies reported in literature tend to focus solely on energy demand and load forecasting (Seyedzadeh et al., 2018); the novelty of this study is the evaluation of the integrated system as a whole. The inclusion of non-linear internal dynamics and feed-back loops from building control and Energy Management System (EMS)s make this a challenging task; for this reason we have selected Support Vector Regression (SVR) models that converge on the optimum solution and are designed to better handle non-linearity as standard linear regression techniques. We also present and discuss how to make these models accessible so they can be retrieved and employed in a practical time frame.

\section{Simplified Mathematical Modelling}

Mathematical approaches to building simulation can either be steady state or transient depending on the level of detail required. Steady state modelling is generally faster as it neglects time constants and assumes the building is in equilibrium with the environment. For example the BREDEM model neglects the impact of thermal capacitance by averaging the temperature over the daily cycle to calculate an average heat output (Anderson, 2002). This does not capture 
the capacitance effects of the building materials and is more suited to applications where low resolution results are acceptable, for example when reporting at monthly or annual intervals. Transient simulations take into account the thermal dynamics of the building and energy system and therefore are more suited for generating demand profiles at high resolutions, for example at time steps between 1 second and 1 hour.

\section{Building Envelope}

In its simplest form, the transient heat transfer of a building envelope can be modelled as a resistorcapacitor network of temperature nodes representing air volumes and building materials. In this approach, the nodes have properties such as mass, temperature, thermal resistance and thermal capacitance. There is no limit to the number of nodes; however the nodes of complex models can be lumped together to form a simplified model. The verification of a two-node lumped model showed close agreement to an explicit solution solved using the finite difference approach (Kampf 2007) The simplification of envelope models have been implemented in Modelica (Baetens et al., 2015) (Lauster et al., 2014). In the latter it was found that a low order model, based on guidelines in the VDI 6007, generated acceptable results compared with an IDA ICE model. The same secondorder model was compared to a reference model comprised of detailed components from the same library and achieved an $88 \%$ decrease in simulation speed while maintaining comparable results (Lauster et al., 2014). While showing promise for the simulation of building envelopes, these studies do not consider the influence of coupling with an energy system.

\section{Energy System}

The primary purpose of the energy system is to provide a comfortable and functional internal environment for the building occupants. Energy systems are generally comprised of a generator, a distribution network, storage and an emitter. The more components in system, the greater number of equations and the longer it will take to simulate. To simplify the simulation in hydraulic systems, the terms for pressure can be neglected, which makes it easier to calculate the mass and energy balances across each component. Stinner et al. developed an enthalpy connector that works on this principle; using this connector they achieved a reduction in simulation speed of nearly $5 \mathrm{x}$ when compared to the standard Modelica.Fluid package (Stinner et al., 2015). A demonstration of a large detailed building model in Modelica, comprised of 32 detailed building zones, multiple integrated energy technologies and various controllers, took 18 hours to solve a one-year simulation (Jorissen et al., 2015). This indicates that reliance on these libraries alone will not be sufficient at achieving the target simulation of this project.

\section{Reduced Order Modelling}

Reduced Order Models (ROMs) are simplifications of dynamical models which consist of many equations and variables. They are commonly used to simulate complex processes within a practical amount of time, yet maintaining acceptable accuracy. ROMs are created using Model Order Reduction (MOR), a process which identifies the essential features of the complex original system. To produce the ROM, the original model needs to be simulated for a short period, during which the MOR approach captures the essential features of the system. ROMs have a particular relevance to Computational Fluid Dynamics (CFD) problems which are now part of commercial packages (Ansys, 2017). Mullen et al. extracted ROMs from CFD simulations of a multi-zonal building to enable the building management system to make rapid decisions based on the impact of changing parameters. Using the ROMs, they were able to generate a solution in 20 seconds compared to 5-8 hours for the CFD simulation. Despite this, the models still required a converged initial solution from which the ROM would be extracted. A parametric investigation revealed that the ROMs produced acceptable results if the initial conditions varied by $5^{\circ} \mathrm{C}$ (Mullen et al., 2015). However there is no discussion on the impact of changing other parameters e.g. air flow rate, internal gains etc. as these could invalidate the ROM. Kim and Braun used the Balanced Truncation Method to reduce the number of states in a thermal network based on finite volume formulation (Kim and Braun, 2015). Only the building envelope was considered because Heating, Ventilation and Air Conditioning (HVAC) systems introduce non-linearity. Using this approach, a 100x speed reduction was achieved compared to TRNSYS. In a separate study Shi and O'Brien used orthogonal decomposition to capture the first order thermal result before using a clustering method to group zones together (Shi and O'Brien, 2016). The method achieved a $95.6 \%$ reduction in computational time compared to the original model. The parameter dependence and time taken to prepare the ROM make this approach impractical for the rapid evaluation of integrated energy systems

\section{Machine Learning}

Machine learning techniques differ from ROMs as they make predictions when exposed to new data. ROMs on the other-hand are a simplification of a well-defined problem, and are therefore highly sensitive to changes in the input parameters. Machine learning models also suffer from this problem but they allow greater control over the parameters used in the training phase and are able to capture nonlinearity. Typical applications for machine learning include stock market prediction, weather forecasting and targeting marketing. Machine learning has also been used to predict energy consumption and 
performance. Gao and Malkawi used an unsupervised, K-means clustering approach to predict the overall energy demand of a building as an alternative benchmarking technique for non-commercial buildings (Gao and Malkawi, 2014). Interestingly, in the feature selection stage, they found that the most influential features of the building were those related to operational factors rather than construction materials and heating unit type. Zhang and O'Neill used multiple machine learning techniques to predict the hot water demand profile using outside dry bulb air temperature as the sole input value (Zhang et al., 2015). Despite the seemingly limited selection of input variables, they were able to predict demand with a respectable RMSE of 11.60-12.24. The authors suggest that inclusion of the occupant schedules would have a significant influence on the model prediction in some cases.

\section{Time Series Prediction}

Time series prediction is a field of statistical methods that can be either univariate or multivariate. A popular univariate technique is Autoregressive Integrated Moving Average Model (ARIMA). ARIMA is a linear regression method that examines past correlations in data to predict future outputs. ARIMA can also be adopted to account for temporal variation or seasonality. Despite the popularity of ARIMA, many problems, such as building performance simulation, are non-linear with multiple influential observations. The general aim of a multivariate time series prediction can be expressed as (1).

$$
\hat{x}\left(t+\Delta_{t}\right)=f\left(x\left(t-v_{1}\right), x\left(t-v_{2}\right), \ldots,\left(t-v_{n}\right)\right)
$$

Where $\hat{x}$ is the predicted value of a discrete time series $x$ and $v_{1}$ to $v_{n}$ are the input variables (Sapankevych and Sankar, 2009).

Artificial Neural Network (ANN) is a machine learning technique, inspired by the neural connections inside a brain, that approximates a mapping function between the input and output variables. Young Tae Chae used an ANN to predict sub-hourly electricity usage in commercial buildings. In this study the key input variables were day-type indicator, time-of-day, HVAC set temperature, ambient dry bulb temperature and outdoor humidity. The study was able to predict the daily electricity demand, at 15-min resolution forecasting interval with 10\% Root Mean Square Error (RMSE) (Chae et al., 2016).

Kandananond used a hybrid approach using ARIMA and an ANN to predict the electricity consumption of Thailand. The ANN took multiple input variables (SET index, GDP and Export) to determine the output energy. The ANN outperformed the ARIMA approach; however the improvement was not deemed to be significant and it was concluded that the ARIMA method is preferable due to its practicality Kan- dananond (2011).

Time series prediction can also be achieved using Support Vector Machine (SVM)s. The concept behind SVM is to find a function of the input values which deviates from the measured output by no greater than the specified error term. For this reason the SVM, unlike ANN, is guaranteed to converge to the optimal solution(Sapankevych and Sankar, 2009). The application of SVM to time series estimation is called support vector regression (SVR). This is an extension of linear time series estimation based on linear regression. In SVR a nonlinear kernel basis (such as radial basis functions) is used to better capture nonlinearities in the estimated time series.

\section{Method}

In this project we have used Modelica for detailed simulations of the performance of buildings with coupled energy systems. The results from these detailed simulations are then used to train a library of models, which aim to predict a performance profile faster, and without a significant loss of accuracy, compared to the detailed simulation model. The stages of the approach are as follows:

1. Creation of the envelope component using archetype data

2. Connection of systems inside the Modelica model using a Python wrapper

3. Simulation of Modelica model in JModelica to generate energy profiles

4. Training of an SVR model using the simulation results

5. Prediction of a demand profile from new input data using the trained model

\section{Envelope Archetypes}

The Episcope Tabula Project was an EU funded initiative that used a consistent classification scheme to create building typologies across the partner countries Loga et al. (2016). The Tabula database defines typical construction properties of external wall, internal wall, roof, floor and window for different age bands in each of the participating countries. In this project the Tabula archetypes for single family houses in Germany were used to create the set of input parameters required by the MixedAir thermal zone of the LBNL Buildings Modelica Library. A summary of these parameters are displayed in Table 1.

\section{Connecting Systems}

The majority of components used in this project are from the Modelica Buildings Library developed by the Lawrence Berkeley National Laboratory (LBNL) (Wetter et al., 2015). This is because the library is compatible with JModelica and the building zone models within the library have been validated against the BESTEST standard. 
Table 1: Summary of the properties for each archetype. The values were derived from the DE.N.SFH.Gen.ReEx archetype of the Tabula dataset.

\begin{tabular}{lll}
\hline $\begin{array}{l}\text { Age } \\
\text { Category }\end{array}$ & $\begin{array}{l}\text { Gross } \\
\text { Area } \\
{\left[\mathrm{m}^{2}\right]}\end{array}$ & $\begin{array}{l}\text { Building } \\
\text { U-Value } \\
{\left[\mathrm{W} / \mathrm{m}^{2} \mathrm{~K}\right]}\end{array}$ \\
\hline$<1859$ & 245.62 & 2.40 \\
\hline $1860-1918$ & 190.40 & 1.50 \\
\hline $1919-1948$ & 336.80 & 1.42 \\
\hline $1949-1957$ & 121.60 & 1.30 \\
\hline $1958-1968$ & 160.90 & 1.14 \\
\hline $1969-1978$ & 193.92 & 0.82 \\
\hline $1979-1983$ & 207.04 & 0.71 \\
\hline $1984-1994$ & 164.48 & 0.49 \\
\hline $1995-2001$ & 136.74 & 0.36 \\
\hline $2002-2009$ & 153.25 & 0.29 \\
\hline $2010-2015$ & 264.70 & 0.27 \\
\hline
\end{tabular}

A Python wrapper has been created that treats Modelica systems as components. Parameters are specified at the highest level of the system and these are directly accessed using the Python wrapper. The systems are connected together using a look up table, which returns the Modelica script required to connect the two systems.

Table 2: The SVR settings used to train the model.

\begin{tabular}{ll}
\hline Parameter & Value \\
\hline Kernel & Radial-basis function \\
\hline Error Penalty $C$ & 2.0 \\
\hline Inverse Kernel Bandwidth $\gamma$ & 2.0 \\
\hline Epsilon $\epsilon$ & 0.01 \\
\hline
\end{tabular}

\section{Model Training}

When training the model it is vital to control causality between the training inputs and outputs. This could pose a problem when dealing with the many different parameter combinations. In SVMs the input and output parameters can be controlled. The SVR class from the Scikit-learn Python Package was used to train the models. The SVR training requires the tuning of hyper-parameters as shown in Table 3 . A preliminary study was carried out to compare the performance of SVR against standard linear regression. Radiative heat prediction RMSE sensitivity to U-value are shown in Figure 1 show the radiative heat RMSE prediction for SVR and linear regression for buildings with three different construction years (1918, 1950, 1980). Nominal U-Value for each building is represented by a relative value of 1.0 , for which the prediction error is minimal. The SVR is able to capture the influence of the U-Value while the linear regression is not able. SVR performance is also better than linear regression for U-Value within $\pm 5 \%$ of its nominal value. Variation of RMSE versus relative $\mathrm{U}$-Value is represented as an indication of its the sen- sitivity. The 1980 is less sensitive to variation of the U-Value.

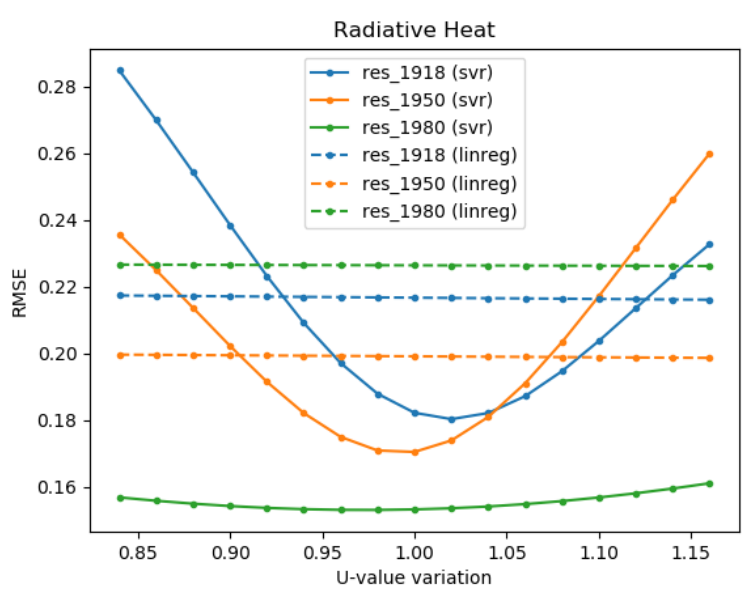

Figure 1: Sensitivity analysis to U-Value. Radiative heat RMSE prediction for SVR and linear regression and three different archetypes. Nominal U-Values are represented by a relative value of 1.0 .

The SVR hyper-parameters settings used in this study are shown in Table 2 .

\section{Case Studies}

In this paper we report on three different case studies where this approach has been applied:

- Case 1: Prediction of internal temperature and heating load for a single zone building under different climates. A model was trained from simulated data using weather parameters to predict the annual profiles of the heating load and internal air temperature of the building.

- Case 2: Prediction of heating demand profile from archetype data. In the first study, 20 days of training data was used to predict the performance over the subsequent 10 days for each of the archetypes. A model was trained using simulated energy demand profiles simulated from nine of the eleven archetypes. The trained model was then used to predict the specific heating load $\left(W / m^{2}\right)$ profile for the remaining two unseen archetypes.

- Case 3: Prediction of radiative and convective output from a PVT system installed in buildings of different construction materials.

\section{Results}

\section{Case 1}

The simulation used to train the model contained a single thermal zone connected to a controller that heated the zone when its temperature fell below 20 ${ }^{\circ} \mathrm{C}$. There was no cooling system present. The model was used to predict the internal temperature and heat demand using climate variables as inputs. The prediction was validated against a simulation created 
Table 4: Computation time and accuracy metrics for the one year simulation, training and prediction phases for temperature (summer) and heating (winter) using 15 min resolution.

\begin{tabular}{lll}
\hline Phase & Temp. & Heating \\
\hline 1 year simulation & \multicolumn{2}{c}{$332 \mathrm{sec}$} \\
\hline Seasonal training & $42 \mathrm{sec}$ & $39 \mathrm{sec}$ \\
\hline Seasonal prediction & $21 \mathrm{sec}$ & $25 \mathrm{sec}$ \\
\hline Coefficient of determination & 0.71 & 0.73 \\
\hline RMSE & $3.23^{\circ} \mathrm{C}$ & $2.50 \mathrm{~kW}$
\end{tabular}

from the same inputs. It was found that the support vector algorithm had difficulty handling periods of no heat demand during the summer, and the controlled internal zone temperatures during the winter. As a result, using a whole year of input data for both predictions lead to $10 \mathrm{x}$ increase in training and prediction time, and a loss in accuracy. For this climate and building type, it was known that heating is not required in the summer and the internal temperature is controlled in the winter; therefore predictions of heat demand and zone temperature were only made in the winter and summer respectively. The weather parameters used to train the models included irradiance (diffuse and direct), wind speed and ambient temperature (dry bulb, wet bulb and dew point). The results of the prediction are shown in Figure 2 and a comparison of simulation, training and prediction times are shown in Table 4.

\section{Case 2}

This case is made up of two sub-studies, in both a model was trained to predict the heating demand of an archetype using building U-Value, percentage of south facing wall and exposed perimeter. These parameters were identified as having the largest influence on the cumulative heating energy demand of the building using a correlation matrix. In the first study, 20 days of training data was used to predict the performance over the subsequent 10 days, the RMSE of heating power for each archetype is shown in Figure 4. In the second study, one month of training data from 9 archetypes was used to predict the heating and cooling demand for two other archetypes for the same month of March. The RMSE of this prediction is
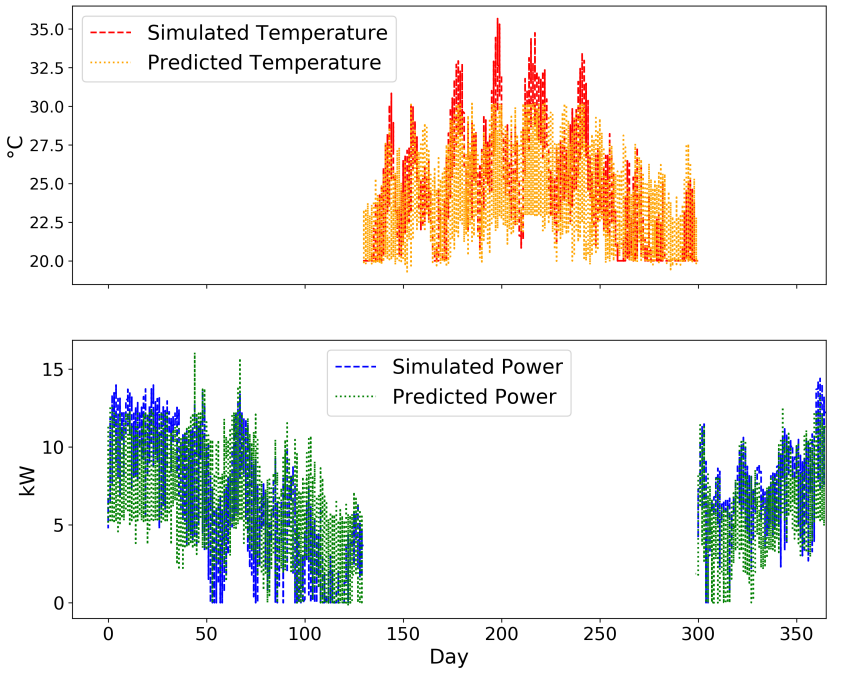

Figure 2: A comparison of simulated and predicted zone temperature (top) and heating load (bottom). Temperature is predicted during the summer months and heating load during the cooling months.
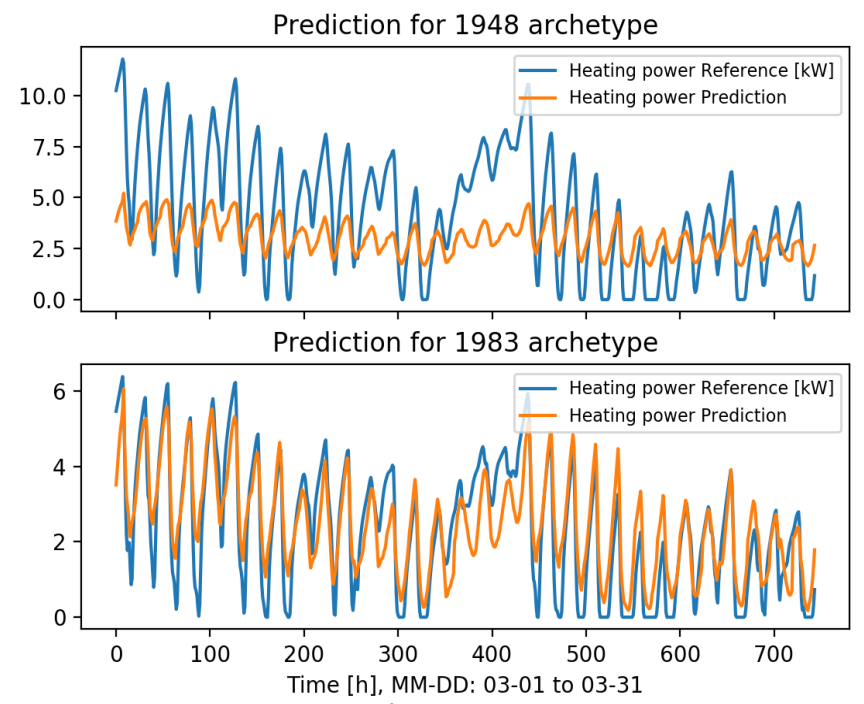

Figure 3: Prediction of heating power using building archetypes. In this case, 9 archetypes are used for training, while prediction is done for two 2 other archetypes. RMSE are shown in Figure 5.

Table 3: Intuitive rules for SVR hyper-parameters tuning.

\begin{tabular}{lll}
\hline Hyper-parameters & Increased value $\nearrow$ & Decreased value $\searrow$ \\
\hline Kernel Bandwidth $\frac{1}{\gamma^{2}}$ & Wide kernel bandwidth & Narrow kernel bandwidth \\
& Filtering/smoothing effect & Sharp and spiky effects \\
\hline Regularization $\frac{1}{C}$ & Better robustness to & May lack robustness towards \\
& unpredictable dynamics. & unpredictable dynamics. \\
& Less fitting accuracy. & Better fit on the training data set. \\
& Avoiding overfitting. & Risk of overfitting. \\
\hline Epsilon $\epsilon$ & Larger insensitive tube & Smaller insensitive tube \\
& around data. & around data. \\
\hline
\end{tabular}


shown in Figure 5. A comparison of the simulated and predicted profiles are shown in Figure 3.

In these plots, the trained RMSE is the RMSE when the model is re-fed with data that was used in the training. The prediction RMSE is the RMSE of input data not used to train the model, but used to make predictions. For all models, the RMSE is compared with the average heating demand of heat property to determine the deviation.

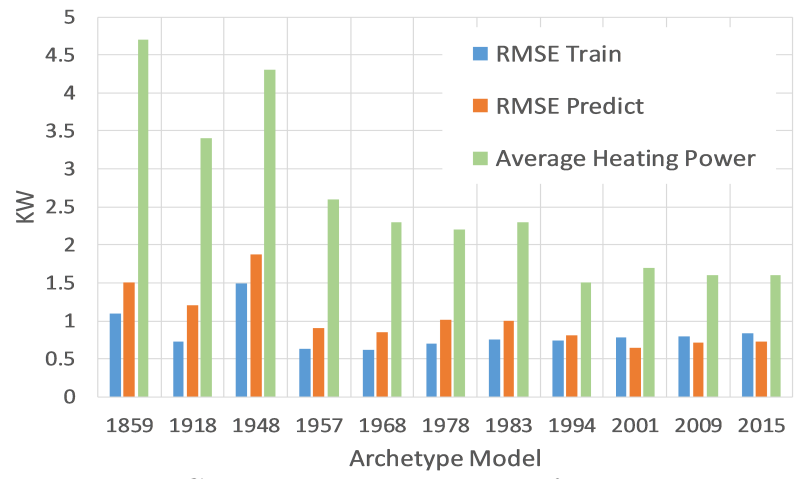

Figure 4: Case 2.1: Prediction of Heating power RMSE [kW] using 20 days of training and prediction for the following 10 days using all 11 archetypes. Average heating power for March is also displayed.

\section{Case 3}

A model was trained to predict the radiant and convective output of a pre-sized PVT system. The input to the model were the building's area-weighted U-Value and weather parameters: global horizontal irradiance and ambient dry bulb temperature. One of the main challenges was to capture the offset between the in heat transfer after peaks of irradiance.

The U-Values input parameters, corresponding to three different building archetypes, referenced by construction year, are used to train the model on the same time period, e.g. April 1st to 20th as shown in Figure 6. Then, the prediction is done on the period of April 21th to April 30th for these three different U-Values to evaluate their ability to distinguish between the three archetypes. The overall input/output signals used for training and prediction phase, along with reference signals, are shown in Figure 6.

\section{Discussion}

The trained models were able to predict a the summer temperature profile and winter heating demand in 21 and 25 seconds respectively, compared to 332 seconds for the simulation. The difference in total energy demand between the simulated and predicted model was $0.12 \%$ despite the profiles not having an exact match, see Figure 2 with a RMSE of $2.5 \mathrm{~kW}$. This first study represented a relatively simple case, where seven weather parameters were used to predict two outputs, zone air temperature and heating power. Using this approach achieved a prediction speed of

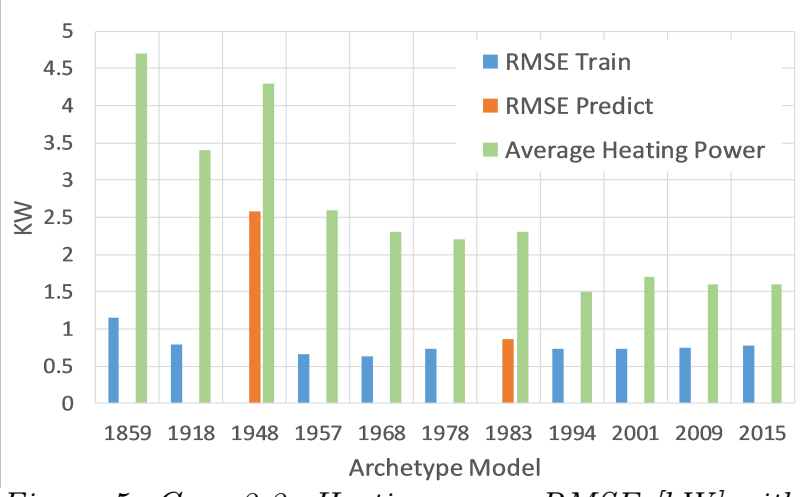

Figure 5: Case 2.2: Heating power RMSE [kW] with training on 9 archetypes and prediction on 2 other archetype on the all month of March. Average heating power for March is also displayed. The time series of the prediction is shown in Figure 5.

over 10x faster than the simulation with comparable values for overall energy use. However care must be taken when using this approach. In this case, the omission of predicting heating loads in the summer, and controlled internal temperatures during the winter, was an obvious decision; however in other cases the decision might not be so clear. Attempting to predict variables with insufficient input data, or the inclusion of bogus dynamics, will lead to long training and prediction times.

In the second study archetype data was used to create a trained model. Such a trained model would be appropriate when there is a need to simulate the performance of a building with a configuration unseen before, provided it is with the parameter limits used to create the trained model. In this study the model was trained using building u-value, exposed perimeter, percentage of total wall area for each window orientation, global irradiance and ambient dry bulb temperature. As the room was connected to a temperature controller for both heating and cooling, it was deemed unnecessary to predict the zone temperature as this would simply fluctuate between the two set-points. This study used eight input parameters to predict two outputs, heating power and cooling power. In the ten day projection, the model achieved a RMSE of $1.6 \mathrm{~kW}$ for heating and a RMSE of 0.22 $\mathrm{kW}$ for cooling. The cooling prediction was not effective as the training data used was from the heating season, highlighting the importance of selecting the relevant parameters during the training phase. When the model was used to predict the performance of an archetype outside of the training data, the simulation time for the three month period was on average $43 \mathrm{sec}$ for the archetypes compared to a training and prediction time of $15 \mathrm{sec}$ and $2.3 \mathrm{sec}$ respectively.

In the third study the performance of a fixed size PVT system was applied to buildings of the different construction materials (represented by the year of con- 

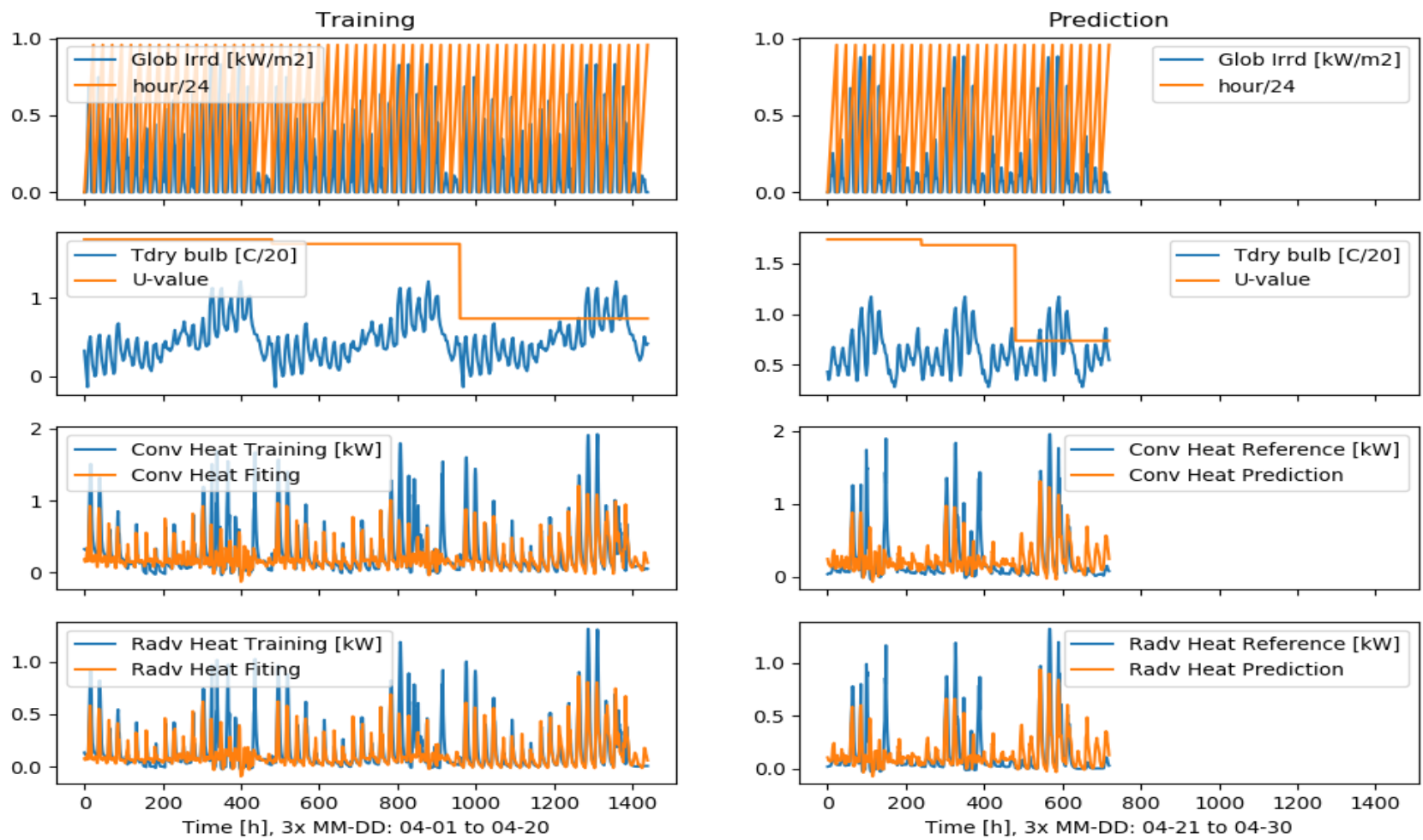

Figure 6: Training and prediction of the heating load (convective and radiative) of the building with PVT system. Three different U-Values, corresponding to three different building archetypes, are used to train the model. Prediction RMSE of radiative heat and its sensitivity to U-Value is shown in Figure 1 - Study 3.

struction). The value of this study is to understand the ability of the trained model to capture non-linear dynamics within a system of multiple-feed back loops and controllers. The PVT system contained pump controllers that switch the system off when there is insufficient radiation. The collector was connected to a hot water cylinder which delivered hot water to radiators when the temperature of the room fell below $20{ }^{\circ} \mathrm{C}$. There was no back-up system and the model predicted the convective and radiative exchanges with the zone through the radiator system. Two months of training data was used to predict the subsequent months. The results showed that it was possible to predict the radiative and convective exchange with a RMSE of $170 \mathrm{~W}$ and $270 \mathrm{~W}$ respectively, and comparison to their respective total energy exchange differed by $4.5 \%$ (radiative) and $3.5 \%$ (convective).

\section{Conclusion}

In this study SVR was successfully able to decrease the time needed to generate an energy profile of a building that would otherwise be produced using detailed simulation. As with other reduced-order modelling approaches, a detailed model is needed to generate the data to train the model; however, once trained, this model can predict an energy profile much faster than the detailed simulation. The cases described in this paper show that these predictions can be made using both weather and archetype data as inputs to the trained model. The method was also able to predict the convective and radiative exchange of a PVT system with the thermal zone of the building; thus showing that the method can effectively capture non-linear dynamics from internal feedback loops within the system. In all three studies the choice of input parameters has proven vital in ensuring quality of the prediction.

Further work is required to investigate the sensitivity of these models to changing parameters, which will involve applying these methods to more complex integrated systems. Another future challenge of this research is to define the parameter limitations of each trained model so that they can be applied practically to a given problem.

\section{Acknowledgements}

This work is funded by Eturnity AG and The Swiss Innovation Agency - Innosuisse.

\section{Nomenclature}

$\hat{x} \quad$ predicted value

$v_{n} \quad$ input variable $n$

$x \quad$ time

\section{Abbreviations}

ANN Artificial Neural Network. 3 
ARIMA Autoregressive Integrated Moving Average Model. 3

BPS Building Performance Simulation. 1

CFD Computational Fluid Dynamics. 2

EMS Energy Management System. 1

HVAC Heating, Ventilation and Air Conditioning. 2

IBPSA International Building Performance Simulation Association. 1

LBNL Lawrence Berkeley National Laboratory. 3

MOR Model Order Reduction. 2

RMSE Root Mean Square Error. 3

ROM Reduced Order Model. 2

SVM Support Vector Machine. 3

SVR Support Vector Regression. 1

\section{References}

Anderson, B. R. (2002). Bredem-12 model description, 2001 update. BRE. Garston, UK.

Ansys (2017). Reduced-Order Modelling How and Why An Overview. www.ansys.com .

Baetens, R., R. De Coninck, F. Jorissen, D. Picard, L. Helsen, and D. Saelens (2015). OpenIDEAS an Open Framework for Integrated District Energy Simulations. In BS2015, pp. 347-354.

IBACOS, Inc., Pittsburgh, PA (United States) (2011). Strategy Guideline. Accurate Heating and Cooling Load Calculations.

Chae, Y. T., R. Horesh, Y. Hwang, and Y. M. Lee $(2016,1)$. Artificial neural network model for forecasting sub-hourly electricity usage in commercial buildings. Energy and Buildings 111, 184-194.

Eturnity (2019). Our solutions for digital consulting of heating systems. www.eturnity.ch/en/heating/.

Gao, X. and A. Malkawi $(2014,12)$. A new methodology for building energy performance benchmarking: An approach based on intelligent clustering algorithm. Energy and Buildings 84, 607-616.

IBPSA (2017). International Building Performance Simulation Association Project 1. https://ibpsa.github.io/project1.

Jorissen, F., M. Wetter, and L. Helsen (2015). Simulation speed analysis and improvements of Modelica models for building energy simulation. In Proceedings of the 11th International Modelica Conference, Versailles, France, September 21-23, 2015, Number 118, pp. 59-69.
Kandananond, K. (2011, 8). Forecasting Electricity Demand in Thailand with an Artificial Neural Network Approach. Energies 4(8), 1246-1257.

Kim, D. and J. E. Braun (2015, 11). A general approach for generating reduced-order models for large multi-zone buildings. Journal of Building Performance Simulation 8(6), 435-448.

Lauster, M., M. Brüntjen, H. Leppmann, M. Fuchs, J. Teichmann, R. Streblow, and D. Müller (2014). Improving a Low Order Building Model for Urban Scale Applications. In BauSIM 2014, pp. 511-518.

Lauster, M., J. Teichmann, M. Fuchs, R. Streblow, and D. Mueller (2014). Low order thermal network models for dynamic simulations of buildings on city district scale. Building and Environment 73, 223231.

Loga, T., B. Stein, and N. Diefenbach (2016, 11). TABULA building typologies in 20 European countries - Making energy-related features of residential building stocks comparable. Energy and Buildings 132, 4-12.

Mullen, D. T., M. M. Keane, M. Geron, and R. F. Monaghan (2015, 7). Automatic extraction of reduced-order models from CFD simulations for building energy modelling. Energy and Buildings 99, 313-326.

Sapankevych, N. and R. Sankar $(2009,5)$. Time Series Prediction Using Support Vector Machines: A Survey. IEEE Computational Intelligence Magazine 4(2), 24-38.

Seyedzadeh, S., F. P. Rahimian, I. Glesk, and M. Roper $(2018,12)$. Machine learning for estimation of building energy consumption and performance: a review. Visualization in Engineering $6(1), 5$.

Shi, Z. and W. O'Brien (2016). Building Energy Model Reduction using Principal Component Analysis and Affinity Propagation Clustering of Thermal Zones. In Asim-IBPSA Asia Conference.

Stinner, S., M. Schumacher, K. Finkbeiner, R. Streblow, and D. Müller (2015). FastHVAC - A library for fast composition and simulation of building energy systems. In Proceedings of the 11th International Modelica Conference, pp. 921-927.

Wetter, M., M. Bonvini, T. S. Nouidui, and W. Zuo (2015). Modelica buildings library 2.0. In Building Simulation 2015, Hyderabad, India.

Zhang, Y., Z. O'Neill, B. Dong, and G. Augenbroe $(2015,4)$. Comparisons of inverse modeling approaches for predicting building energy performance. Building and Environment 86, 177-190. 